\title{
VORTEX RINGS - EXPERIMENTS AND NUMERICAL SIMULATIONS
}

IULIA - RODICA DAMIAN - PhD Student, Reorom Laboratory, "Politehnica" University of Bucharest, Power Engineering Faculty, e-mail: rodi_damian@yahoo.com

NICOLETA OCTAVIA TĂNASE - Assistant, PhD, Reorom Laboratory, "Politehnica" University of Bucharest, Power Engineering Faculty, e-mail: notanase@yahoo.com

ȘTEFAN - MUGUR SIMIONESCU - PhD Student, Reorom Laboratory, "Politehnica" University of Bucharest, Power Engineering Faculty, e-mail: stefan_simionescu@yahoo.com

MONA MIHĂILESCU - Lecturer, PhD, "Politehnica" University of Bucharest, Physics Department,

e-mail: mona.mihailescu@yahoo.com

\begin{abstract}
The present paper was concerned with the experimental study of the time evolution of a single laminar vortex ring generated at the interface between water and isopropyl alcohol. The experiment was performed by the submerged injection of isopropyl alcohol in a water tank of $100 \times 100 \times 150 \mathrm{~mm}$. A constant rate of $Q_{0}=2 \mathrm{ml} / \mathrm{min}$ was maintained using a PHD Ultra 4400 Syringe Pump with a needle having the inner diameter $D_{0}=0.4 \mathrm{~mm}$. The dynamics of the vortex formation was recorded with a Photron Fastcam SA1 camera at 1000 fps equipped with an Edmund Optics objective VZM1000i. The numerical simulations were performed on a 2D geometry using the ANSYS-FLUENT code with the Volume of Fluid multiphase model and the viscous-laminar solver. The numerical flow patterns were found to be in good agreement with the experimental visualizations.
\end{abstract}

Keywords: flow visualization, isopropyl alcohol, buoyancy, CFD.

\section{Introduction}

Vortex rings are one of the most fundamental and interesting phenomena and have fascinated many authors, starting from the classical work of H. Helmholtz [1] up to nowadays scientists.

The practical applications of the vortex ring formation are found in the field of hydrodynamics such as flow control, fluid mixing, heat transfer and propulsion, but also in the biological and biomedical field like animal locomotion or internal flows.

Almost all the previous research was focused on vortex rings generated by immersed jets using the same liquids or following up the impact of buoyancy acting on the same direction as the momentum $[1,5,6,9,12,14]$.

A. B. Olcay and P.S. Krueger [8] used the same fluid for both the impinging jet and the ambiental one. Other representative experiments, such as those in which the ejected fluid was more dense, with upper or lower injection were conducted by Camassa et al. [14], and O. J. Myrtroeen and G. R. Hunt respectively [12]. An example regarding the injection from the bottom of the tank of a less dense fluid than the ambiental one was analyzed by D. Bond and H. Johari [9].

The original contribution of our research is emphasized by the vortex ring formation imposing the buoyancy to act in the opposite direction of the momentum, as seen in Fig.1. However this subject of studies remains one of the most important in the fluid mechanics field.

Buoyant intrusions are common in natural and engineered systems like volcanic eruptions, piston engine fuel injection and microscale heat exchange in microelectromechanical systems [2]. 


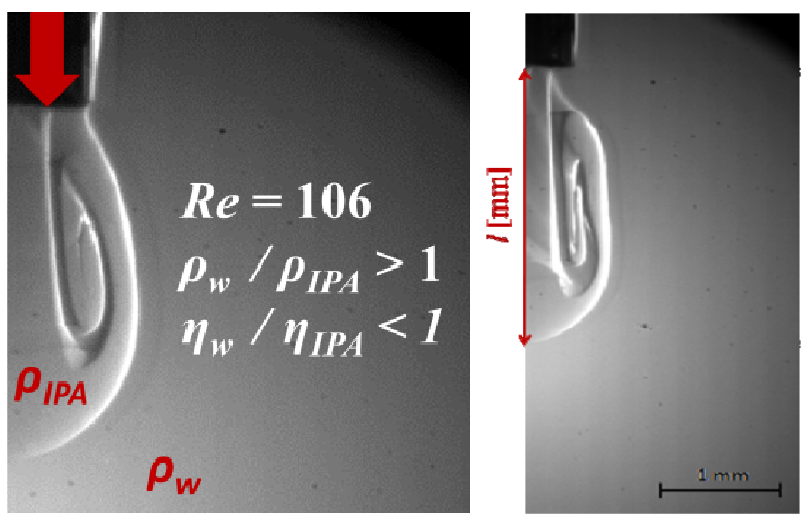

Fig.1 - The analyzed case

The aim of this article is to perform a numerical and experimental study on the dynamics of vortex rings. Qualitative direct visualizations of the flow were created by injecting a less viscous and dense fluid than the ambiental one, from the top of the tank, in order to observe the influence of buoyancy upon their formation and characteristics. The numerical simulations were performed in a 2D geometry with the laminar solver implemented in FLUENT, using the VOF code for the calculation of the interface between the two liquid phases.

Theoretical, numerical and experimental researches about the vortex rings have characterized the movement as a function of dimensional parameters: radius $R$, core size $a$, circulation $\Gamma$, and non-dimensional parameters $[3,4,5]$ :

- The formation number, $L / D_{0}$, where $L$ is the stroke length and $D_{0}$ is the inner orifice diameter,

- The Reynolds number, $R e=\rho \cdot \mathrm{V}_{0} \cdot D_{0} / \eta$, where $\mathrm{V}_{0}$ is the velocity of the injected fluid.

In laboratory, vortex rings are typically generated by forcing a slug of fluid through a tube or orifice. The vortex sheet on the fluid slug rolls up into a vortex core [9]. Consequently, both ejected fluid, which comes from the noozle, and ambient fluid, which is pulled from the vicinity of the needle outlet, must be accelerated as the ring forms. The structure of the vortex ring immersed in the tank, generated by the jet is shown in Fig. 2.

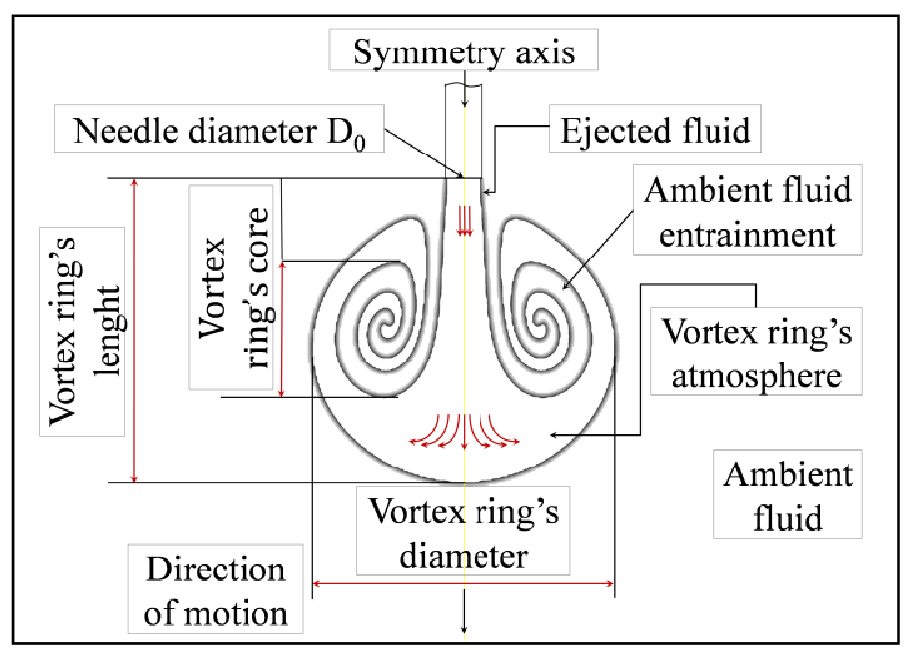

Fig.2 - The structure of the vortex ring immersed in the tank

The shape and size of a vortex ring depend on the ratio between the radius of cross section of the ring core and the ring radius $n=a / R$. For thick vortex rings, $n>0.0116$, the atmosphere of the vortex represents a deformed sphere, for $n=0.0116$ the atmosphere of the vortex ring in its cross section represents a "figure of eight" and finally for thin vortex rings, $n<0.0116$, the atmosphere of the vortex ring represents a torus embracing the thin core of the vortex ring [2]. 
The circulation that a vortex ring could attain is finite. There is a maximum amount of fluid vorticity that could be contained within a ring [7]. Initially, it was found that for values smaller than $L / D_{0} \approx 4$, a solitary vortex ring was formed, while for larger values of $L / D_{0}$, a leading vortex followed by a trailing jet and secondary vortices were observed. The morphology changes from a shape resembling an oblate hemisphere, to a mushroom-shaped structure.

Further investigations revealed that the formation number may depend on various factors and the studies showed that the ratio $L / D_{0}$ may reach values up to 8 .

Buoyant vortex rings form a different class of vortices where the density difference between the ring and ambient fluid profoundly alters the flow dynamics [9]. A buoyant jet can be broadly classified as positively buoyant, if the buoyancy force and momentum fluxes are aligned in the same direction or negatively buoyant if the buoyant force and momentum flux are opposed [13].

\section{Experimental results}

The experiment was performed by the injection of isopropyl alcohol in a water tank of $100 \times 100 \times 150 \mathrm{~mm}$ at a constant flow rate of $2 \mathrm{ml} / \mathrm{min}$, obtaining vortex rings with lower density than the ambient fluid.
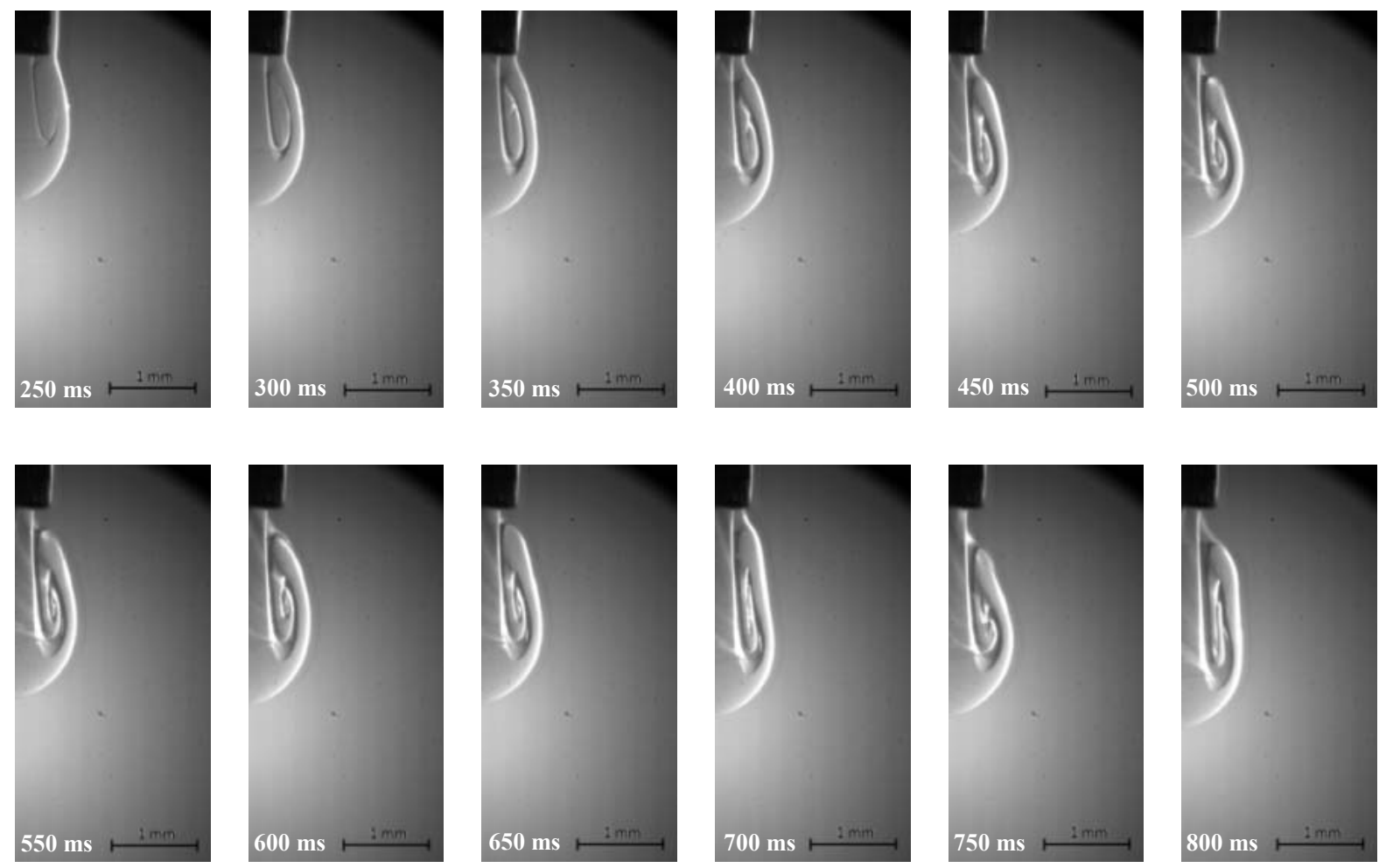

Fig.3 -Vortex ring evolution

The characteristic parameter of the flow was the Reynolds number of 106 . The flow was maintained constant using a PHD Ultra 4400 Syringe Pump with a needle having the inner diameter $D_{0}=0.4 \mathrm{~mm}$. The dynamics of the vortex formation was recorded with a Photron Fastcam SA1 camera at 1000 fps equipped with an Edmund Optics objective VZM1000i, as shown in Fig. 4. 


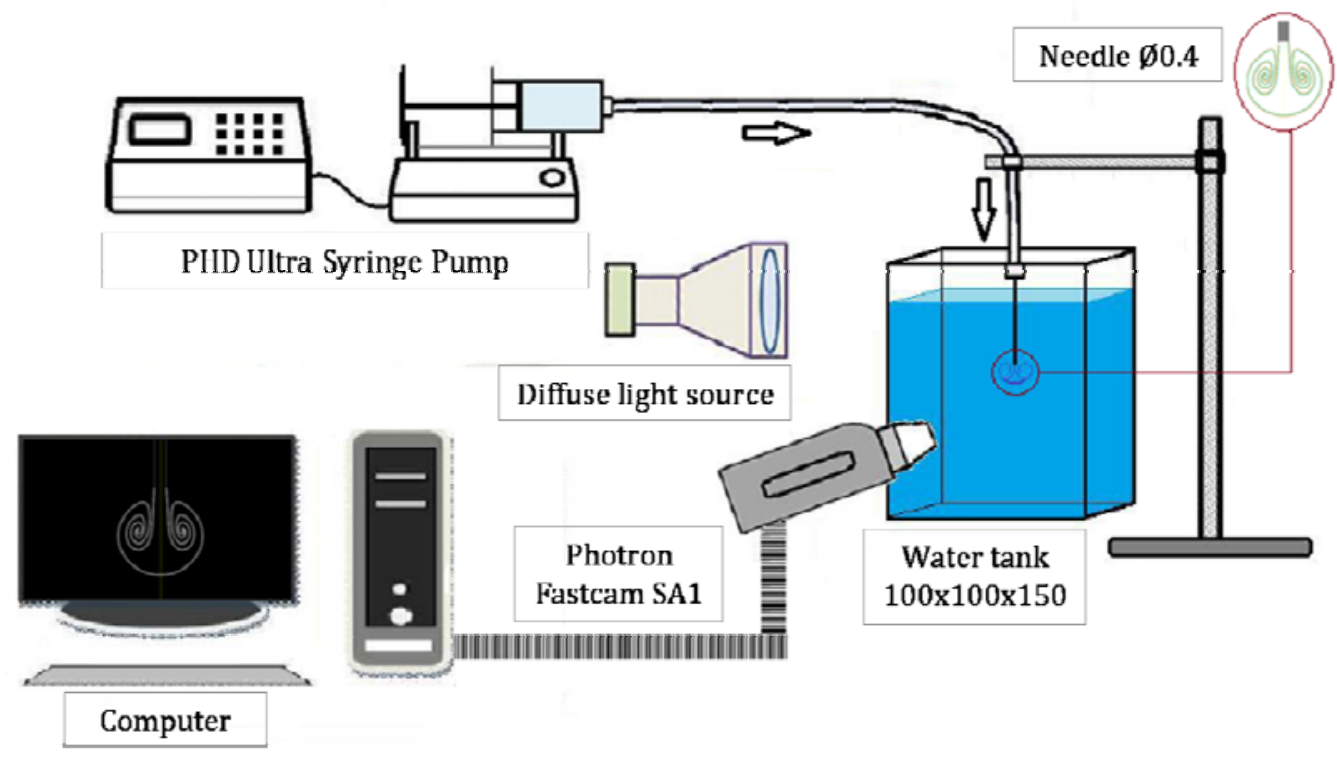

Fig.4 - Experimental setup: Harvard Apparatus 4400 Ultra Syringe Pump, PhotronFastcam SA1, Edmund Microscopic Objective VZM1000i, glass tank, flat needle tip with inner diameter $0.4 \mathrm{~mm}$.

The working fluids have the following properties: the densities of isopropyl alcohol $\rho_{I P A}=$ $786 \mathrm{~kg} / \mathrm{m}^{3}$ and water $\rho_{w}=1000 \mathrm{~kg} / \mathrm{m}^{3}$. The viscosities are $\eta_{I P A}=2.5 \mathrm{mPa} \cdot \mathrm{s}, \eta_{w}=1 \mathrm{mPa}$. $\mathrm{s}$ respectively.

After the PHD Ultra 4400 Pump was turned on and started to inject the fluid, the stream of isopropyl alcohol slowly separated and started to roll-up. The ejected fluid entrained the ambient fluid, and it was observed a symmetrical buoyant vortex ring with an elongated structure. In figure 3 are presented the experimental results for the shape of the vortex ring at different time steps.

By analizing the length of the obtained vortical structures, we observed a time dependence, with three different behaviours. Initially, the vortex ring shape was dominated by the inertia force. It followed a transition period after which the buoyancy force became preponderent, as observed in Fig.5.

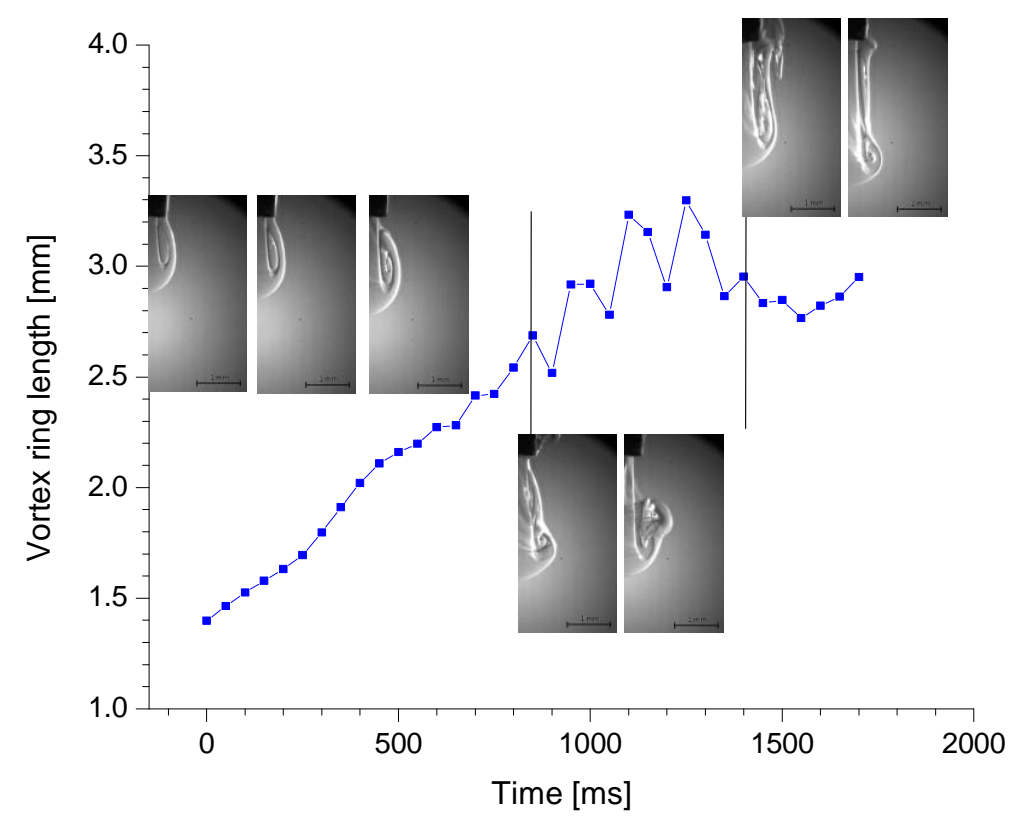

Fig.5 -Vortex length evolution, 1 (measured from the tip of the needle to the vortex lower boundary). 


\section{Numerical results}

The aim of the numerical study was to establish the shape of the vortex rings, according to the experiments. The numerical computations of the vortex ring were performed using the VOF model implemented in FLUENT, the mixture of water and isopropyl alcohol being solved simultaneously with the same laminar solver in a 2D geometry. In the VOF model of mixture, the fluids were considered incompressible and immiscible (no real diffusion between the isopropyl alcohol and water being allowed), see for details [13]. The separation interface was obtained by connecting the cells of equal volume fraction using the Geo - Reconstruct scheme. From the computational point of view, Fluent solves the equation system consisting of the continuity equation, the movement equation, and the mass conservation equation of the immiscible fluid in motion.

To achieve the numerical calculation using the FLUENT code, a geometry that reproduces the actual flow was built and then a discretization of the domain into a spatial grid ("mesh") consisting of finite elements.

The construction of the geometry and mesh of the flow field have a great influence on the obtained results. For the present study, the construction of the flow field and its mesh were carried out using the pre-processor Gambit 2.4, which provides a common set of CAD functions for creating domains, and features implemented specifically for rapid creation of predefined and structured geometries.

The appearance and development of the vortex ring at the interface between isopropyl alcohol in water was followed using the Multiphase module of ANSYS-FLUENT. The VOF multiphase model can be used for two or more immiscible fluids, aiming to determine the surface between the two fluids by solving a single set of motion equations for all phases of fluid occupying the whole field analyzed.

For the approximation of the interface between two phases $(a, b)$, FLUENT calculates at first the volume fraction of the fluid $a$ (noted $\alpha_{a}$ ) present in each part of the domain, thus setting whether or not the respective fluid occupies the volume element:

$$
\left\{\begin{array}{c}
\alpha_{a}=0 \rightarrow \text { the volume element doesn't contain the fluid } a \\
\alpha_{a}=1 \rightarrow \text { the fluid } a \text { fills the volume element entirely } \\
0<\alpha_{a}<1 \text { the volume element contains the interface between } a \text { and another fluid phase }
\end{array}\right.
$$

In order to determine the interface between the two phases, the code solves a particular solution of the equation of continuity for the determination of the volume of fluid fraction, which contains the mass transfer between the two phases $\left(\dot{m}_{b a}, \dot{m}_{a b}\right)$. This solution has the following form:

$$
\frac{1}{\rho_{a}}\left[\frac{\partial}{\partial t}\left(\alpha_{a} \rho_{a}\right)+\nabla \cdot\left(\alpha_{a} \rho_{a} \mathbf{v}_{a}\right)=\sum_{p=1}^{n}\left(\dot{m}_{b a}-\dot{m}_{a b}\right)\right]
$$

The volume fraction equation can be solved explicitly or implicitly, based on the calculation method used to determine the interface.

In the studied case of the vortex ring formation, more work areas were used. The mesh of the flow domain (with 309967 elements and 311151 nodes) was made using quadrilateral elements. All simulations were performed using the same mesh and have run on a 64-bit Quad-core computer with 16 GB RAM memory.

The initial distribution of the phases and the corresponding boundary conditions are shown in figure 6: (i) at the entrance was the first phase, isopropyl alcohol with uniform and constant velocity $\left(\mathrm{v}=\mathrm{v}_{0}\right)$, (ii) the flow took place at atmospheric pressure, at the outlet the condition was $p=p_{0}$; and (iii) adherence conditions on the wall: $\mathrm{v}=0$. 


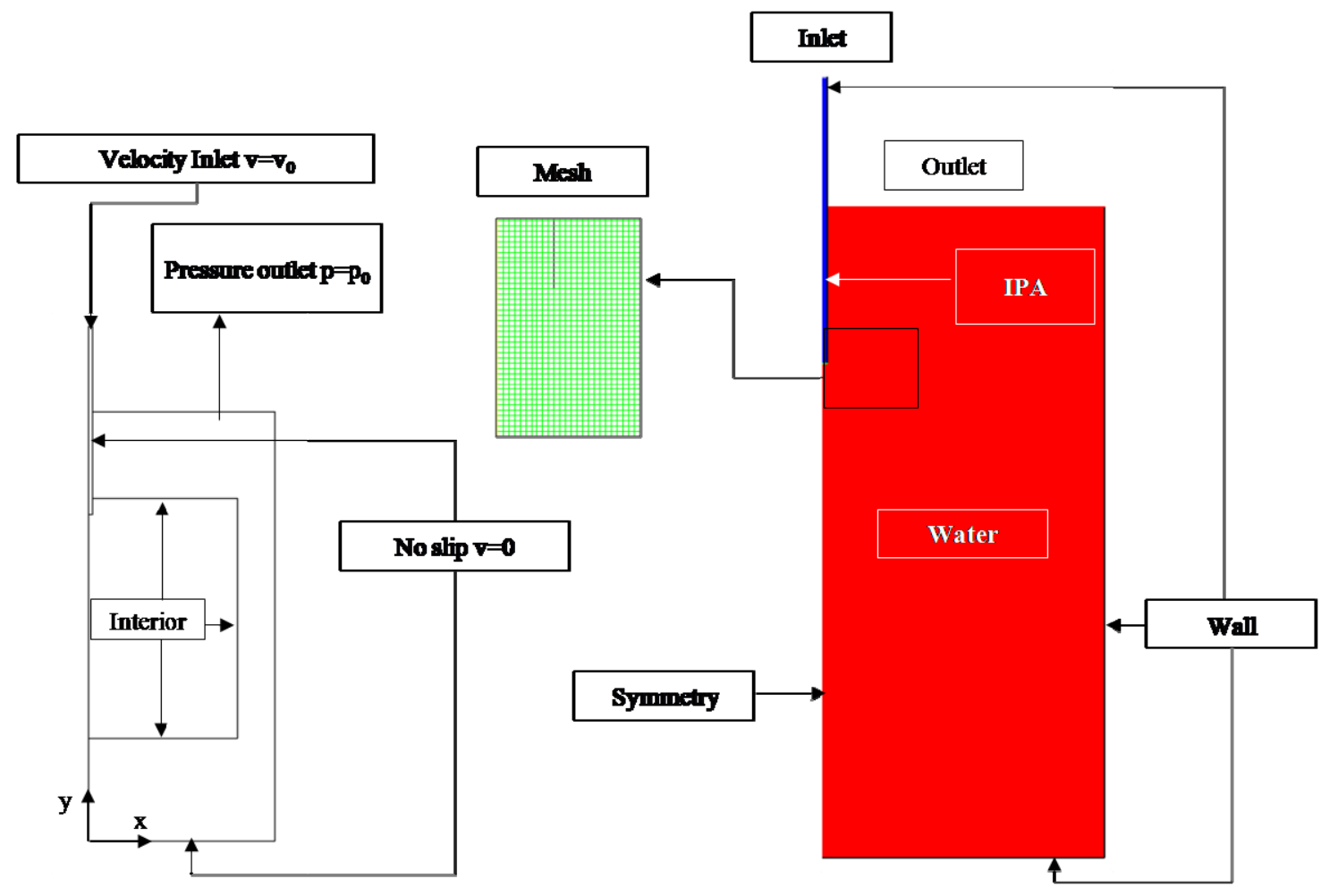

Fig.6 - Numerical working domain, initial phases configurations and the boundary conditions implemented in the code FLUENT; detail of the structured mesh in vicinity of the needle.

In figure 7 are presented the results for the shape of the vortex rings corresponding to the laminar model and at the different time steps.
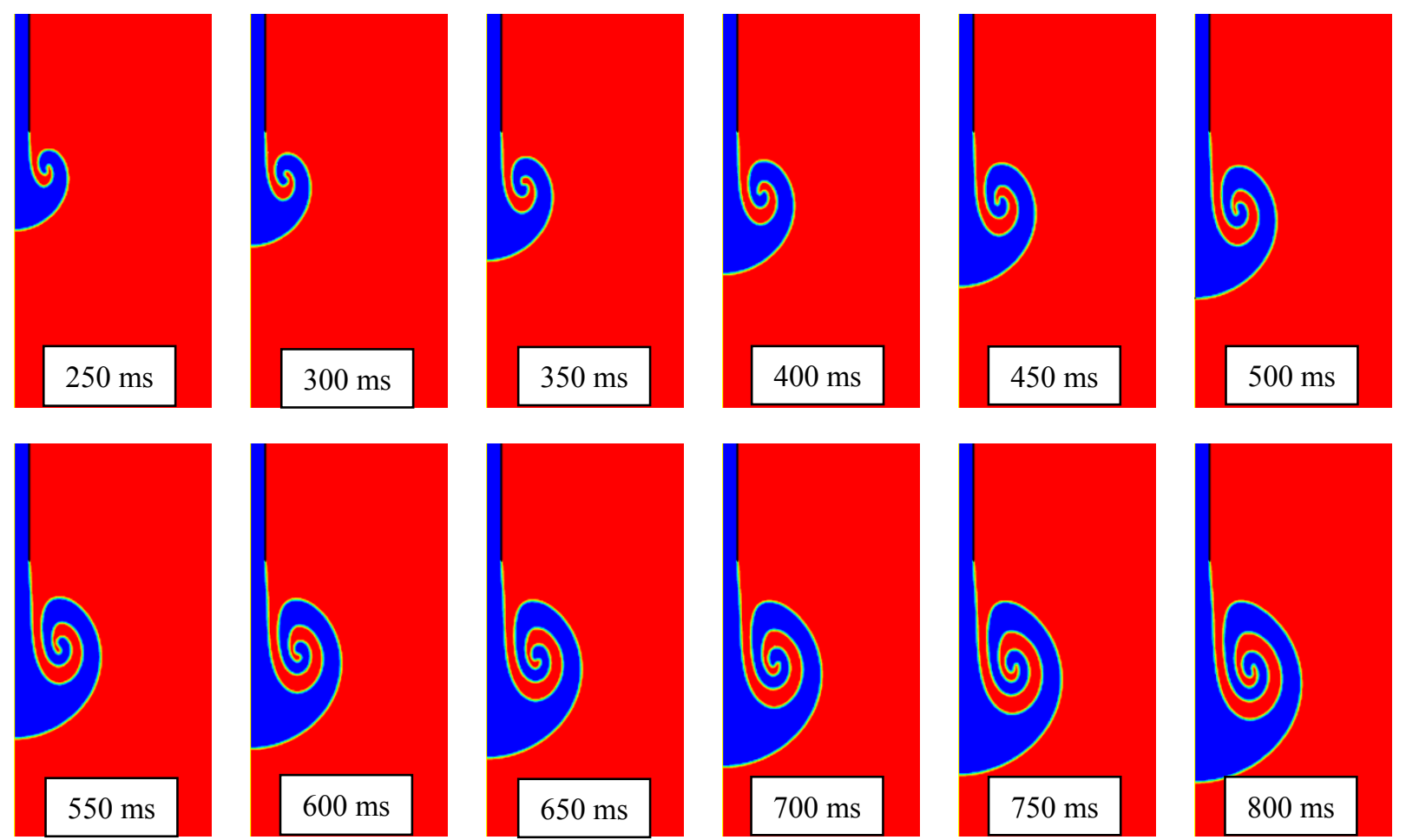

Fig.7 - Numerical simulations of vortex ring evolution 


\section{Comparison of experimental with numerical results}

The Target was to establish the experimental procedure and to correlate the results with the numerical simulations.

We analized first the numerical results, by representing the vortex length evolution in time and we obtained the curves for different Re numbers. After wich we compared them with the values of this same parameter, again at different Re numers $(48<R e<106)$. We performed several simulations for a series of velocities, because we wanted to observe the quantitative data. There are obvious differences and one reason may be the fact that between the 2 fluids wasn't considered the surface tension and diffusion at the interface, the buoyancy force isn't preponderent.

In figure $8 \mathrm{a}$ are presented the results for the length of the vortex rings corresponding to the laminar model at different time steps and $R e$ numbers, while in figure $8 \mathrm{~b}$ it is shown the comparison of the numerical results with the experimental ones.
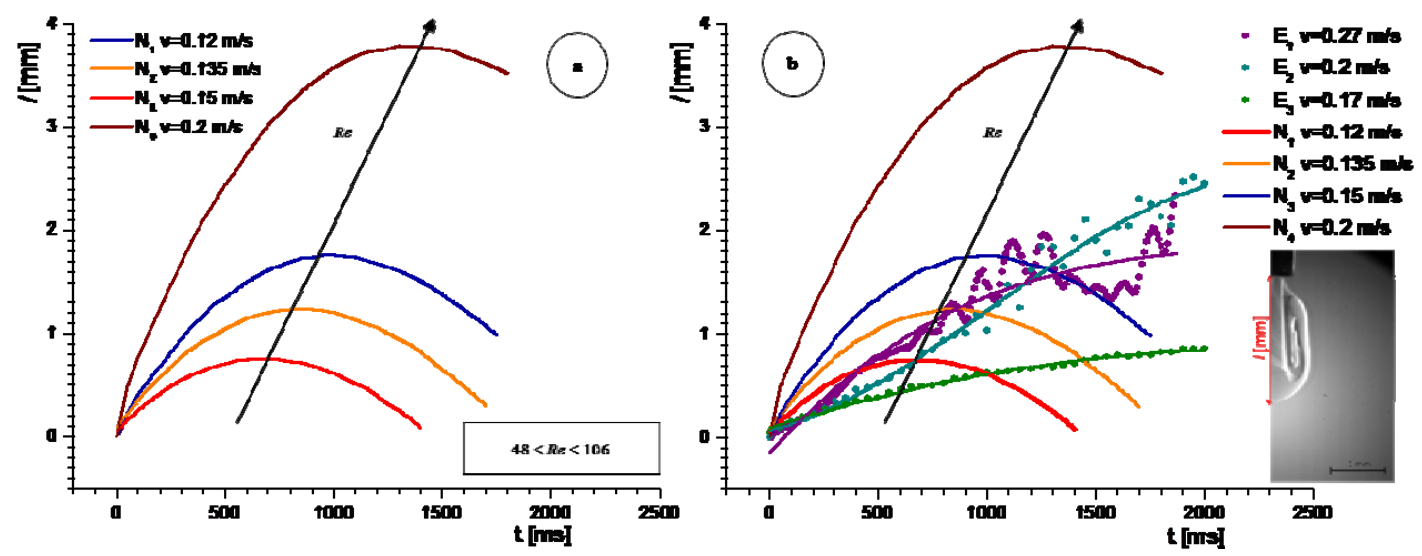

Fig.8 - Vortex length evolution 1 (measured from the tip of the needle to the vortex lower boundary); 8a numerical results of the length of the vortex rings corresponding at the different time steps and $R e$ numbers; $8 \mathrm{~b}$ the comparison of the numerical results with the experimental ones for various inlet velocities.

\section{Conclusions}

The paper was dedicated to the experimental and numerical investigations of the vortex rings formation with the buoyancy acting on the opposite direction as the momentum, due to the difference in density and viscosity of the chosen two working fluids.

The numerical flow patterns were found to be qualitatively in good agreement with the experimental manifestations.

The experimental investigations were limited up to now only to the direct visualization of the vortex rings. The author's intentions to progress with this study is to obtain the quantitative data of velocities, so to compare directly the experimental results with the numerical simulations.

In the future the numerical model will be perfected by testing various tipes of meshes, taking under consideration the diffusion coefficients between the two fluids, performing also experiments with other fluids and of course creating a 3D model.

The present result is very promising and offers possibilities to develop in the future more detailed studies of the vortex rings. 


\section{Acknowledgement}

Iulia - Rodica Damian's work has been funded by the Sectoral Operational Programme Human Resources Development 2007-2013 of the Ministry of European Funds through the Financial Agreement POSDRU/159/1.5/S/132397.

The work of Ștefan - Mugur Simionescu and Nicoleta - Octavia Tănase has been funded by the Sectoral Operational Programme Human Resources Development 2007-2013 of the Ministry of European Funds through the Financial Agreement POSDRU/159/1.5/S/132395.

The authors acknowledge the support and advice of professor Corneliu Bălan (Reorom Laboratory, "Politehnica" University of Bucharest, Power Engineering Faculty).

This paper was presented at EENVIRO 2014 Conference.

\section{References}

[1]. Sullivan I. S., Niemela J. J., Hershberger R. E., Bolster D., Donnelly R. J. (2008). Dynamics of thin vortex rings, Cambridge University Press, Journal of Fluid Mechanics, vol. 609, pp. $319-347$

[2]. Wang R.J., Wing-Keung Law A., Adams E.E., Fringer O. B. (2009). Buoyant formation number of a starting buoyant jet, Theoretical Physics of Fluids.

[3]. Meleshko V. V., Gourjii A. A., Krasnopolskaya T. S. (2012). Vortex rings: history and state of the art. Journal of Mathematical Sciences, vol. 42, No. 6, December.

[4]. Akhmetov D. G. (2001). Formation and basic parameters of vortex rings, Journal of Applied Mechanics and Technical Physics, vol. 42, No. 5.

[5]. Maxworthy T.(1977). Some experimental studies of vortex rings, Journal od Fluid Mechanics, vol. 81, part. 3.

[6]. Laursen T. S., Rasmussen J. J., Stenum B., Snezhkin E. N. (1997). Formation of a 2D pair and its 3D breakup: an experimental study, Experiments in Fluids.

[7]. Palacios-Morales C., Zenit R. (2013). Vortex ring formation for low Re numbers, Acta Mechanica.

[8]. Olcay A. B., Krueger P. S. (2010). Momentum evolution of ejected and entrained fluid during laminar vortex ring formation, Theoretical Computational Fluid Dynamics.

[9]. Bond D., Johari H. (2010). Impact of buoyancy on vortex ring development in the near field, Experiments in Fluids.

[10]. Mohseni K., Ran H., Colonius T. (2001). Numerical experiments on vortex ring formation, Journal of Fluid Mechanics.

[11]. Nitsche M., Krsny R. (1994). A Numerical study on vortex ring formation at the edge of a circular tube, Journal of Fluid Mechanics.

[12]. Myrtroeen O.J., Hunt G.R. (2010). Negatively bouyant projectiles - from weak fountains to heavy vortices", Journal of Fluid Mechanics.

[13]. Wang R.J., Wing-Keung Law A., Adams E.E. (2011). Pinch - off and formation number of negatively bouyant jet, Phisics of Fluids.

[14]. $\quad * * *$ Fluent Inc., Fluent 6.3 user's guide, 2006.

[15]. Camassa R., Khatri S., McLaughlin R., Mertens K., Nenon D., Smith C., Viotti C. (2013). Numerical simulations and experimental measurements of dense-core vortex rings in a sharply stratified environment, Computational Sicence and Discovery. 